\title{
REUMATOLOGÍA
}

Sociedad Argentina de Reumatología

\section{casos clínicos y revisión de la literatura}

\section{Quistes óseos de localizacion inusual como causa de lumbalgia crónica en adultos}

\author{
Maldonado, F.N.; Vergel Orduz, L.; Brigante, J.A.; Dubinsky, D. \\ Servicio de Reumatología del Sanatorio Güemes, Ciudad Autónoma de Buenos Aires
}

\section{Palabras clave:}

Lumbalgia, quiste óseo, quiste óseo simple, quiste óseo unicameral, quiste óseo aneurismático

\section{Key words:}

Low back pain, bone cyst, simple bone cyst, unicameral bone cyst, aneurysmal bone cyst

Mail de contacto:

fedemaldonado87@gmail.com

\section{RESUMEN}

Se presenta el caso de un paciente con quistes en el hueso iliaco como causa atípica de lumbalgia crónica a la cual se puede enfrentar el reumatólogo, y se hará una revisión de la literatura sobre los tipos de quistes óseos y sus diagnósticos diferenciales. También se hará mención de sus opciones de tratamiento

\section{ABSTRACT}

The case of a patient with cysts in the iliac bone is presented as an atypical cause of chronic low back pain that the rheumatologist may face, and a review of the literature on the types of bone cysts and their differential diagnoses will be made. Mention will also be made of your treatment options

\section{Introducción}

La lumbalgia es una manifestación cuyos diagnósticos diferenciales son tan diversos que hace necesario agruparlos bajo ciertas características, como la presencia de banderas rojas, hallazgos clínicos (inflamatorio, mecánico o neurogénico), y tiempo evolutivo (agudo, subaguda o crónico), para poder guiar su estudio etiológico. Un estudio estimo que entre el $70 \%$ y $80 \%$ de las personas padecerán dolor lumbar en algún momento de su vida, con un pico de incidencia entre 45 y 59 años ${ }^{1}$.

La articulación sacro iliaca (SI) es responsable del 13 a 30 $\%$ de todas las causas de dolor lumbar bajo², dentro de las que sobresalen las enfermedades comprendidas en el grupo de las espondiloartropatías seronegativas. Por este motivo, los pacientes con lumbalgia crónica llegan a la consulta del reumatólogo, quién debe tener la suficiente competencia para evaluar y descartar las variadas patologías que lo pueden generar y así llegar al diagnóstico.

Cuando el dolor persiste por más de 6 semanas, es refractario al tratamiento médico, se acompaña de hallazgos patológicos en el examen físico, o presenta una o más ban- deras rojas, se deberían solicitar exámenes complementarios. Para el caso de patologías que afectan la articulación SI, la fiabilidad del examen físico y la utilidad de la radiografía convencional de pelvis suelen ser bajas ${ }^{3}$, por lo que se requirieren estudios de imágenes más sofisticados como la resonancia magnética (RM) o tomografía (TC).

Se presenta el caso de un paciente con quistes en el hueso iliaco como causa atípica de lumbalgia crónica a la cual se puede enfrentar el reumatólogo, y se hará una revisión de la literatura sobre los tipos de quistes óseos y sus diagnósticos diferenciales. En forma breve se hará mención de sus opciones de tratamiento.

\section{Caso clínico}

Se trata de un hombre de 41 años, sin comorbilidades, que inició hace 10 años con dolor lumbar irradiado en forma intermitente a glúteos, que no lo despertaba por la noche, y se asociaba a rigidez matutina de aproximadamente 1 hora con mejoría tras el ejercicio. Fue tratado con AINEs y kinesiología en repetidas oportunidades, presentando respuesta parcial y transitoria. Se lo derivó al consultorio de 


\section{Figura 1.}
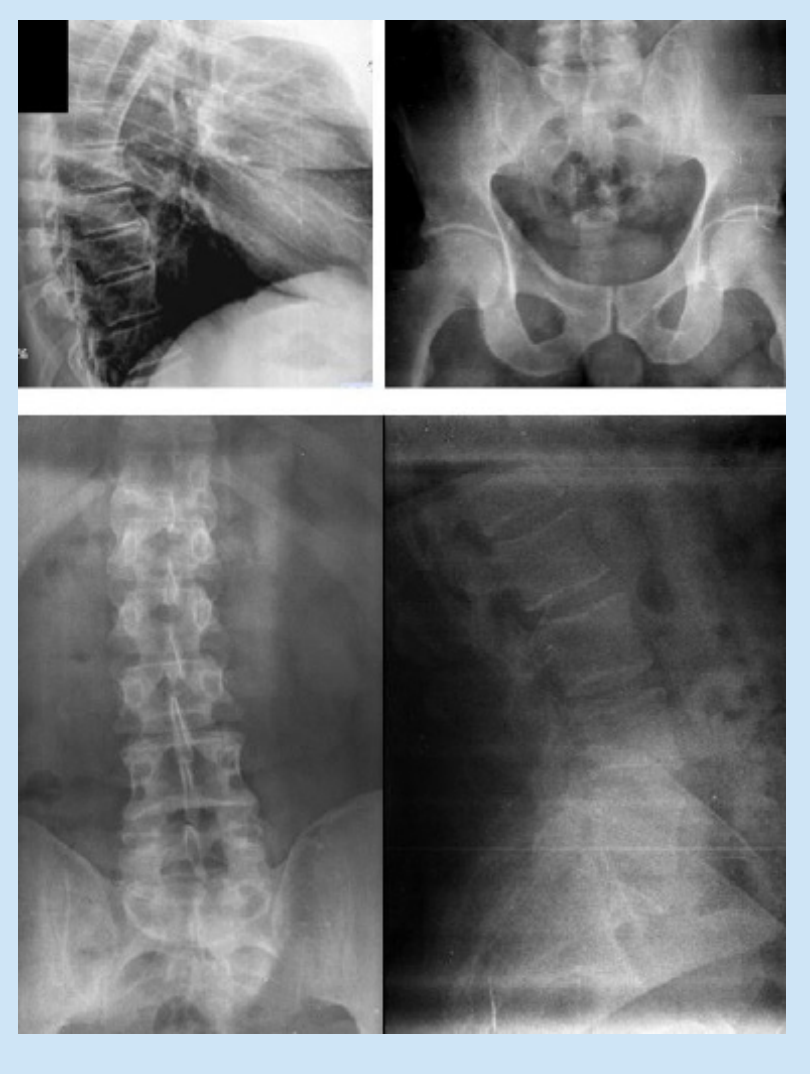

Referencias: a) Rx columna dorsal: calcificación del ligamento longitudinal anterior b) Rx panorámica de pelvis c) Rx columna lumbar frente y perfil.

\section{Figura 2. Clasificación de Enneking y Dunham}

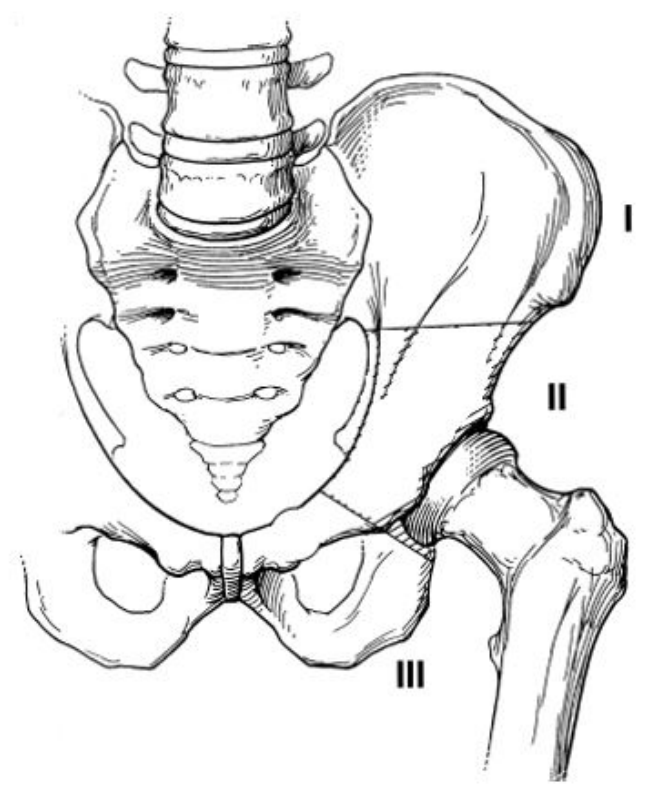

Referencias: Clasificación de Enneking y Dunham para ubicación de lesiones en la pelvis ósea, región I: iliosacral; II, acetabular; III: isquiopubiana. lumbalgia del servicio de reumatología ante la persistencia de su dolencia.

Al interrogatorio dirigido negaba síntomas sistémicos asociados (fiebre, pérdida de peso, sudoración, inapetencia, astenia), antecedentes de uveítis, síntomas gastrointestinales crónicos, antecedentes personales y familiares de psoriasis y de espondiloartropatías.

Al examen físico no presentaba dolor en articulaciones SI, artritis periférica, dactilitis, entesitis, ni lesiones cutáneas o ungueales compatibles con psoriasis. Solamente se constató una leve limitación en la flexión lateral de la columna lumbar, y un engrosamiento nodular del tercio proximal de ambos tendones de Aquiles, que el paciente relacionaba con episodios previos de tendinitis.

Su laboratorio de sangre era normal, sin reactantes inflamatorios elevados, HLA B 27 e inmunoserología negativas (látex $\mathrm{AR}$, y anti CCP). La radiografía (Rx) de columna dorsal mostraba calcificaciones del ligamento longitudinal anterior sobre 3 vertebras contiguas, con espacio intervertebral conservado; la Rx de columna lumbar no presentaba alteraciones (Fig. 1), y la Rx panorámica de pelvis hacia dudar sobre la presencia de alteraciones en las articulaciones SI manifestadas por mayor esclerosis sobre las superficies articulares de ambos huesos iliacos. Finalmente, la RM y la TC de la pelvis evidenciaron lesiones líticas, multiloculadas (septos intraquísticos), adyacentes la articulación SI en ambos huesos iliacos (zona 1 de Enneking y Dunham. Fig. 2), de contenido líquido (hipointenso en T1 e hiperintenso en T2), y presencia del "signo de nivel liquido de doble densidad" en la secuencia T2. El espacio articular se encontraba respetado (Fig. 3 y 4 ).

\section{Discusión}

Los quistes óseos son lesiones líticas de naturaleza benigna, que se presentan habitualmente en la segunda década y generalmente afectan a huesos largos de las extremidades superiores e inferiores. El diagnóstico diferencial debe incluir al quiste óseo simple o unicameral (QU), y al quiste óseo aneurismático (QA).

\section{Quiste óseo Simple o unicameral (QU)}

Es una lesión lítica benigna, usualmente solitaria, de contenido líquido, unicameral o parcialmente septada. Se cree que su formación se debe a estasis venosa dentro del hueso esponjoso, con aumento de la presión y liberación de mediadores inflamatorios, que llevan a la resorción ósea y formación de una cavidad de contenido líquido ${ }^{4-6}$.

Epidemiologia: El 85\% ocurren casi exclusivamente en niños y adolescentes. El pico reportado es entre los 3 y 14 años, con una edad promedio en el momento del diagnóstico de aproximadamente 9 años ${ }^{7-10}$. Los QU representan aproximadamente el $3 \%$ de todos los tumores óseos primarios biopsiados y la relación hombre /mujer es de $2 / 1 .^{10}$

Localización: Involucran principalmente los huesos largos, especialmente el húmero proximal, seguido por el fémur proximal. Estas 2 ubicaciones representan más del $80 \%$ de los $\operatorname{casos}^{4-6}$. Otros lugares son más raros (tibia proximal o diafisaria, húmero diafisario o distal, fémur diafisario o distal, peroné, antebrazo) o excepcionales (metacarpo, falange, huesos del carpo, huesos del pie, escápula, mandíbula). Las lesiones del hueso ilíaco, calcáneo, costillas y radio afectan 
principalmente a adolescentes mayores y adultos ${ }^{4,11-13}$.

Evolución: Los QU generalmente comienzan en la metáfisis de los huesos largos y se desplazan distalmente hacia la diáfisis durante el crecimiento, permaneciendo estáticos o resolviendo completamente ${ }^{4-5}$. Se clasifican como activos cuando están dentro de $1 \mathrm{~cm}$ de la fisis y latentes a medida que avanzan hacia la diáfisis.

En el húmero proximal tienden a desaparecer después de la pubertad. Por el contrario, el 50\% de los pacientes con QU femorales proximales son mayores de 17 años con un rango de edad de hasta 54 años. Alrededor del 6\% al 10\% de los QU se informan en adultos en huesos planos, planteándose la hipótesis de que esta presentación tardía se debe potencialmente a la ubicación del hueso plano que lo protege del trauma ${ }^{14}$.

Los QU son más agresivos en la primera década de la vida y la tasa de recurrencia de estos pacientes es cuatro veces mayor que la de los adolescentes ${ }^{14}$. El QU puede complicarse con un quiste aneurismático secundario.

Manifestaciones clínicas: Pueden ser asintomáticos, siendo un hallazgo en una radiografía solicitada por otras causas (80\%), poco sintomáticos (sensibilidad, tumefacción sobre el hueso afectado) o manifestarse por dolor secundario a fractura patológica ${ }^{5-6}$.

Exámenes complementarios: En las Rx aparecen como lesiones radiolúcidas, bien delimitados por un borde esclerótico, usualmente unicamerales, ubicadas centralmente en la cavidad medular con su eje paralelo al largo del hueso. Puede presentar adelgazamiento del hueso cortical circundante sin reacción perióstica. En caso de fractura, existen 2 hallazgos casi patognomónicos denominados "signo de fragmento caído" (fragmento óseo desplazable dentro del quiste) y "signo de burbuja ascendente" (burbuja de gas que migra hacia arriba) $)^{4-6}$.

La TC ayuda a evaluar localizaciones complejas como la pelvis y columna vertebral, y sirve para evaluar la extensión, grosor de la pared y presencia de complicaciones.

La RM confirma la naturaleza quística de la lesión al evidenciar una señal liquida. La inyección de Gadolinio realza los márgenes y los tabiques o pseudotabiques. En caso de fractura muestra un contenido heterogéneo en todas las secuencias debido a los productos sanguíneos. La reacción perióstica y el edema circundante aparecen hiperintensos en T2 y STIR ${ }^{15,16}$

La gammagrafía ósea y la PET-TC no son contributivas; puede haber normal o hipofijación en quistes no complicados e hiperfijación en caso de fractura. Ocasionalmente se observa un aumento de la actividad de FDG por PET y puede confundirse con metástasis óseas ${ }^{17}$.

La cistografía puede ser diagnostica de QU si la cavidad es ocupada completamente, aunque también podría revelar paredes intraquísticas. Sirve además para evaluar los grados de drenaje venoso ${ }^{4,18}$. El líquido de punción habitualmente es seroso o parduzco, aunque puede ser hemático en caso de fractura previa.

Diagnósticos diferenciales: El diagnóstico diferencial principal es con el QA. Otras lesiones incluidas son la displasia fibrosa, el fibroma no osificante, el granuloma eosinofílico, el encondroma o el fibroma condromixoide ${ }^{4-6}$.

\section{Quiste óseo aneurismático (QA)}

El QA es una lesión benigna, osteolítica, expansiva y llena
Figura 3. TAC de pelvis
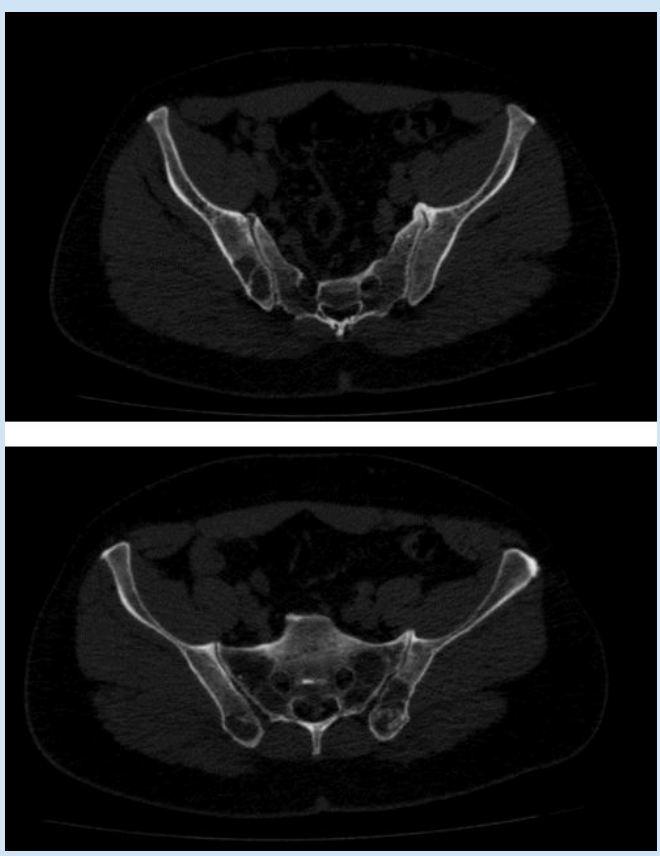

Referencias: Quistes óseos bilaterales con septos internos y delgado margen óseo cortical circundante.

Figura 4. RNM de articulación Sacroilíaca

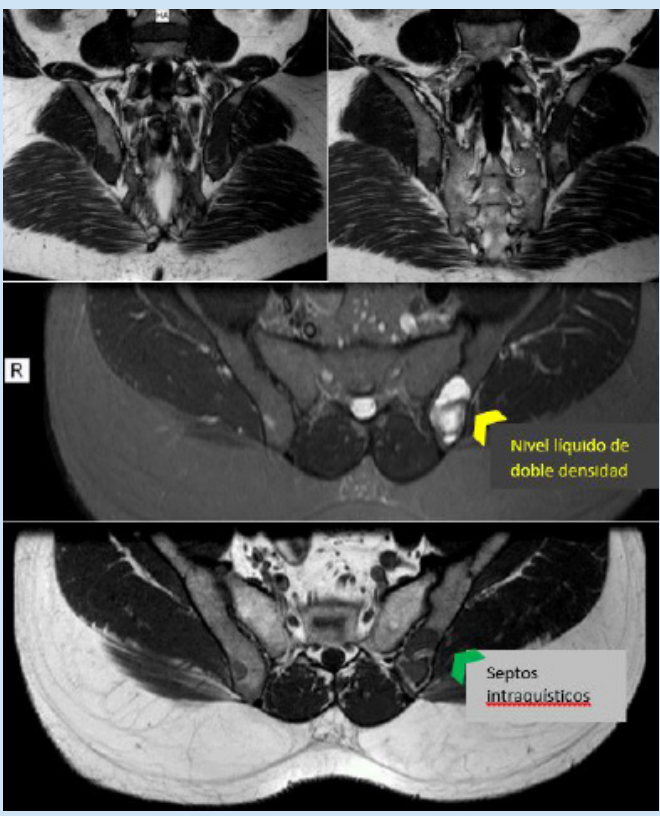

Referencias: Flecha amarilla: Signo del nivel liquido de doble densidad: la capa superior es de alta intensidad de señal, probablemente atribuible al líquido seroso o a la metahemoglobina extracelular. Flecha verde: Septo intraquistico. 
de sangre. El rápido crecimiento de la lesión puede simular malignidad. Pueden ser: primarios (70\% de los casos), asociados a translocaciones cromosómicas del oncogén USP6 ${ }^{19}$; o secundarios ( $30 \%$ de los casos), en reacción a otros tumores óseos subyacentes como tumor de células gigantes, quiste óseo simple, condroblastomas, osteoblastomas, displasia fibrosa, fibroma no osificante y fibromas condromixoides ${ }^{4,20,21}$.

Epidemiologia: Afecta a todas las edades, pero principalmente a personas entre 10-20 años (75-90\% de los casos), con una ligera preponderancia femenina. Comprenden aproximadamente el $1.4 \%$ de todos los tumores óseos ${ }^{4,20,22}$.

Localización: puede involucrar cualquier parte del esqueleto, pero especialmente los huesos largos (67\% de los casos), la columna vertebral (15\%) y la pelvis (9\%). La metáfisis suele estar involucrada, y los huesos más frecuentemente involucrados son el fémur distal, la tibia, el húmero y el peroné. En la columna vertebral, generalmente involucra el arco posterior, principalmente de la zona lumbar, seguido de las vértebras cervicales y luego torácicas. Las lesiones pélvicas con mayor frecuencia involucran el agujero obturador y son periféricas al cartílago trirradiado ${ }^{21}$.

Manifestaciones clínicas: El dolor es el síntoma que más frecuentemente lleva a consultar al paciente. Las lesiones espinales pueden revelarse por dolor, contractura, escoliosis rígida, o más raramente como masa palpable, fractura o síntomas neurológicos ${ }^{4,23}$.

Exámenes complementarios: En la Rx simple, se muestra como una lesión excéntrica, osteolítica, expansiva y algunas veces con finas trabéculas en su interior. La pérdida de los contornos corticales o la extensión a los tejidos blandos puede simular una lesión maligna, lo que indica una forma agre$\operatorname{siva}^{4,21}$. Las lesiones vertebrales involucran el arco posterior o tanto el cuerpo como el arco posterior ${ }^{4,23}$.

La TC permite evaluar la extensión y riesgo de fractura, y es útil en regiones complejas como la pelvis y la columna.

LA RM es el estudio de elección, y es muy útil para diferenciar QU de QA. Un estudio que comparo las características por RM de ambos quistes, determino que el nivel de líquido de doble densidad, la septación y la baja señal en las imágenes T1 y la alta señal en T2 sugieren fuertemente que el quiste óseo en cuestión es un quiste óseo aneurismático, en lugar de un quiste óseo unicameral. La presencia de material de alta señal en ambas secuencias de imágenes sugiere que hay una hemorragia o material coloidal en el quiste ${ }^{24}$. La inyección de gadolinio muestra una mejora de las paredes del quiste y los tabiques internos.

La cistografía demuestra la presencia de tabiques intraquísticos. El líquido de punción es hemático.

Diagnósticos diferenciales: Incluyen el QU, al cual puede asociarse en algunas ocasiones. Otras lesiones incluyen el osteosarcoma telangiectásico, el tumor de células gigantes, granuloma eosinofílico, el osteoblastoma o el tumor maligno, el fibroma no osificante; para los cuales la biopsia podría ser indispensable.

\section{Quistes óseos de la pelvis}

Unni informó afectación del hueso ilíaco en el 8\% de 289 pacientes con quiste óseo aneurismático ${ }^{25}$. Mankin y col. informaron 13 pacientes con lesión pélvica entre 150 pacientes con quistes óseos aneurismáticos ${ }^{26}$. Aycan y col. describieron que el compromiso de la pelvis se dio en menos del $1 \%$ de los 352 casos de QU y 99 casos de $\mathrm{QA}^{13}$. En otro estudio se descri- bió una afectación del hueso pélvico de aproximadamente el $2 \%$ entre los QU. 27,28 .

La porción posterior del hueso ilíaco, adyacente a la articulación SI (Zona 1 de Enneking y Dunham, Figura 2), es un sitio poco frecuente e inesperado para estas lesiones y en general no se describen dentro de los diagnósticos diferenciales de dolor relacionado a la articulación SI. Papagelopoulos y col. informaron que solo 5 de 40 pacientes con QA involucraban esta zona ${ }^{29}$. Otro estudio reporto esta localización en 5 de 16 pacientes con $\mathrm{QU}^{30}$.

\section{Tratamiento}

Ningún tratamiento garantiza la cura definitiva, dado la alta tasa de recurrencias. Para los QU el tratamiento debe ser individualizado de acuerdo al caso. Después de la biopsia del quiste es recomendable esperar 4 a 6 semanas antes de iniciar algún tratamiento, ya que, en algunos casos, el quiste presenta involución espontanea. Las lesiones asintomáticas localizadas en miembros superiores generalmente pueden seguirse con $\mathrm{Rx}$ seriadas y no requerir tratamiento. Las lesiones más grandes con riesgo de fractura, las lesiones sintomáticas y las lesiones de las extremidades inferiores generalmente reciben tratamiento con legrado percutáneo y/o inyección de corticosteroides o de distintos materiales de relleno (hueso desmineralizado, aspirados de medula ósea o sustitutos óseos). Las fracturas patológicas en la extremidad superior generalmente tienen tratamiento conservador. Sin embargo, la fractura inestable o la fractura en áreas que soportan peso como en la extremidad inferior, el tratamiento involucra tanto la fijación de la fractura como el tratamiento del quiste óseo ${ }^{31}$. El enclavado intramedular estable elástico en el caso de huesos largos de niños ha mostrado resultados prometedores ${ }^{32}$. Los conceptos actuales de tratamiento incluyen una combinación de descompresiones, legrado, inyección de corticosteroides/material de relleno y fijación interna en la región que soporta peso ${ }^{33}$.

Para los QA, el legrado quirúrgico y el injerto óseo con o sin terapia adyuvante fue, clásicamente, el modo primario de tratamiento. Varios tratamientos mínimamente invasivos han surgido como una alternativa, incluida la escleroterapia con polidocanol; inyección de corticoides, alcohol absoluto o calcitonia; la embolización selectiva del vaso de alimentación al quiste y la terapia médica con denosumab ${ }^{33-35}$. Las dosis bajas de radiación pueden causar una osificación rápida de la lesión. Sin embargo, es menos utilizado debido al riesgo de transformación maligna.

\section{Conclusión}

El reumatólogo debe conocer el proceso diagnóstico e identificar las banderas rojas del dolor lumbar para guiar la solicitud de exámenes complementarios que permitan alcanzar el diagnóstico. Aunque son un sitio infrecuente de presentación, los diagnósticos diferenciales para las lesiones quísticas en la pelvis deben incluir al QU y al QA. Resulta más probable que por sus características imagenológicas (signo de nivel líquido de doble densidad, septos internos), el caso clínico presentado corresponda a un QA. Será necesaria su pronta intervención para evitar complicaciones como crecimiento expansivo y/o fracturas. 


\section{BIBLIOGRAFÍA}

1. Papageorgiou AC, Croft PR, Ferry S, Jayson MIV, Silman AJ. Estimating prevalence of low back pain in the population. Evidence from the South Manchester Back Pain Survey. Spine 1995; 20:1:889-94

2. Schwarzer AC, Aprill CN, Bogduk N. The sacroiliac joint in chronic low back pain. Spine 1995;20:31-7.

3. Dreyfuss P, Michaelsen M, Pauza K, McLarty J, Bogduk N. The value of medical history and physical examination in diagnosing sacroiliac joint pain. Spine 1996;21:2594- 602

4. Docquier PL. Kyste osseux essentiel. In: EMC. Elsevier Masson SAS; 2011.

5. Cahuzac JP, Sales de Gauzy J. Kyse osseux essentiel. In: Chotel F, Gouin F, editors. Tumeur osseuses bénignes. Paris: Elsevier; 2005. p. 176-87.

6. Kaelin A. Kystes essentiels des os. In: Duparc J, editor. Cahiers d'enseignements de la Sofcot. Paris: L'Expansion scientifique franc, aise; 1995. p. 167-79.

7. Wilkins RM. Unicameral bone cysts, J. Am. Acad. Orthop. Surg. 8 (4) (2000) 217-224.

8. Pretell-Mazzini J, Murphy RF, Kushare I, Dormans JP. Unicameral bone cysts: general characteristics and management controversies, J. Am. Acad. Orthop. Surg. 22 (5) (2014) 295-303.

9. Biermann JS. Common benign lesions of bone in children and adolescents, J. Pediatr. Orthop. 22 (2) (2002) 268-273.

10. Boseker E, Bickel W, Dahlin D. A clinicopathologic study of simple unicameral bone cysts, Surg. Gynecol. Obstet. 127 (3) (1968) 550-560.

11. Kalil RK. Simple bone cyst. In: Fletcher JA, Hogendoorn PCW, Mertens F, editors. WHO tumours of soft tissue and bone. IARC; 2013. p. 350-1.

12. Gentile JV, Weinert CR, Schlechter JA. Treatment of unicameral bone cysts in pediatric patients with an injectable regenerative graft: a preliminary report. J Pediatr Orthop 2013;33(3):254-61

13. Aycan OE, Çamurcu $\triangle Y$, Devrim Ö, Yavuz A, Yavuz SK. Unusual Localizations of Unicameral Bone Cysts and Aneurysmal Bone Cysts: A Retrospective Review of 451 Cases. Acta Orthop Belg 2015;81(2):209-12.

14. Lokiec F, Wientroub S. Simple bone cyst: etiology, classification, pathology, and treatment modalities, J. Pediatr. Orthop. B 7 (4) (1998) 262-273.

15. Wootton-Gorges SL. MR imaging of primary bone tumors and tumor-like conditions in children, Magn. Reson. Imag. Clin. N. Am. 17 (3) (2009) 469-487.

16. Sanal HT, Chen L, Haghighi P, Trudell DJ, Resnick DL. Carpal bone cysts: MRI, gross pathology, and histology correlation in cadavers, Diagn. Interventional Radiol. 20 (6) (2014) 503.

17. Mascard E, Gomez-Brouchet A, Lambot K. Bone cysts: unicameral and aneurysmal bone cyst, J. Orthop. Traumatol.: Surgery \& Research 101 (1) (2015) S119-S127.

18. Ramirez A, Abril JC, Touza A. Unicameral bone cyst: radiographic assessment of venous outflow by cystography as a prognostic index. J Pediatr Orthop B 2012;21(6):489-94.

Fecha de trabajo recibido: 05/01/2021

Fecha de trabajo aceptado: 05/02/2021
19. Warren M, Xu D, Li X. Gene fusions PAFAH1B1-USP6 and RUNX2USP6 in aneurysmal bone cysts identified by next generation sequencing. Cancer Genet. 2017 Apr;212-213:13-18.

20. Cottalorda J, Bourelle S. Aneurysmal bone cystin 2006. Rev Chir Orthop Reparatrice Appar Mot 2007;93(1):5-16.

21. Campanacci M. Aneurysmal bone cyst. Bone and soft tissues tumors. Piccin: Padova; 1999. p. 815-32

22. Nielsen GP, Fletcher JA, Oliveira AM. Aneurysmal bone cyst. WHO classification of tumours of soft tissue and bone. Lyon: IARC; 2013. p. 348-9.

23. Boriani S. Aneurysmal bone cyst of the mobile spine: report on 41 cases. Spine 2001;26(1):27-35

24. Sullivan RJ, Meyer JS, Dormans JP, Davidson RS. Diagnosing Aneurysmal and Unicameral Bone Cysts With Magnetic Resonance Imaging. Clinical orthopaedics and related researc. Number 366, pp. 186-190 01999

25. Unni KK. Dahlin's bone tumors: general aspects and data on 11,087 cases. 5th ed. Philadelphia: Lippincott-Raven; 1996. P. 382-90

26. Mankin HJ, Hornicek FJ, Ortiz-Cruz E, Villafuerte J, Gebhardt MC Aneurysmal bone cyst: a review of 150 patients. J Clin Oncol 2005;23:6756-62.

27. Abdelwahab IF, Hermann G, Norton KI, Kenan S, Lewis MM, Klein MJ. Simple bone cysts of the pelvis in adolescents. A report of four cases. J Bone Joint Surg Am 1991;73:1090-4.

28. Samuelson KM, Momberger GL, Coleman SS. Solitary bone cyst of the ilium. Report of two cases and a review of the literature. Rocky Mt Med J 1975;72:443-5.

29. Papagelopoulos PJ, Choudhury SN, Frassica FJ, Bond JR, Unni KK, Sim FH. Treatment of aneurysmal bone cysts of the pelvis and sacrum. J Bone Joint Surg Am 2001;83- A:1674-81.

30. Hammoud S, Weber K, McCarthy EF. Unicameral bone cysts of the pelvis: a study of 16 cases. Iowa Orthop J 2005;25:69-74.

31. Wilke B, Houdek M, Rao RR, Caird MS, Larson AN, Milbrandt T. Treatment of Unicameral Bone Cysts of the Proximal Femur With Internal Fixation Lessens the Risk of Additional Surgery. Orthopedics. 2017 Sep 01;40(5):e862-e867.

32. de Sanctis N, Andreacchio A. Elastic stable intramedullary nailing is the best treatment of unicameral bone cysts of the long bones in children?: Prospective long-term follow-up study. J Pediatr Orthop. 2006 Jul-Aug;26(4):520-5.

33. Tsagozis P, Brosjö O. Current Strategies for the Treatment of Aneurysmal Bone Cysts. Orthop Rev (Pavia). 2015 Dec 28;7(4):6182.

34. Lange T, Stehling C, Fröhlich B, Klingenhöfer M, Kunkel P, Schneppenheim R, Escherich G, Gosheger G, Hardes J, Jürgens H, Schulte TL. Denosumab: a potential new and innovative treatment option for aneurysmal bone cysts. Eur Spine J. 2013 Jun;22(6):1417-22.

35. Chang CY, Kattapuram SV, Huang AJ, Simeone FJ, Torriani M, Bredella MA. Treatment of aneurysmal bone cysts by percutaneous CT-guided injection of calcitonin and steroid. Skeletal Radiol. 2017 Jan;46(1):35-40. 